\title{
CHANGE DETECTION IN HIGH-RESOLUTION REMOTE SENSING IMAGES USING LEVENE-TEST AND FUZZY EVALUATION
}

\author{
GuangHui Wang ${ }^{1,2,}$, HuaBin Wang ${ }^{2}$, WenFeng Fan ${ }^{2}$, Yu Liu², HuiJie Liu ${ }^{2}$ \\ 1 China University of Mining and Technology, Xuzhou 221116, China-Wanggh@ sasmac.cn \\ 2 Satelite Surveying and Mapping Application,NASG,Beijing 100830,China
}

Commission VI, WG III/7

KEY WORDS: High-resolution images, change detection, Levene-Test, hue-texture features, fuzzy evaluation method

\begin{abstract}
:
High-resolution remote sensing images possess complex spatial structure and rich texture information, according to these, this paper presents a new method of change detection based on Levene-Test and Fuzzy Evaluation. It first got map-spots by segmenting two overlapping images which had been pretreated, extracted features such as spectrum and texture. Then, changed information of all map-spots which had been treated by the Levene-Test were counted to obtain the candidate changed regions, hue information $(\mathrm{H}$ component) was extracted through the IHS Transform and conducted change vector analysis combined with the texture information. Eventually, the threshold was confirmed by an iteration method, the subject degrees of candidate changed regions were calculated, and final change regions were determined. In this paper experimental results on multi-temporal ZY-3 high-resolution images of some area in Jiangsu Province show that: Through extracting map-spots of larger difference as the candidate changed regions, Levene-Test decreases the computing load, improves the precision of change detection, and shows better fault-tolerant capacity for those unchanged regions which are of relatively large differences. The combination of Hue-texture features and fuzzy evaluation method can effectively decrease omissions and deficiencies, improve the precision of change detection.
\end{abstract}

\section{INTRODUCTION}

The remote sensing sensor records the change of the spectral response of two image elements. The acquired multi-temporal remote sensing image is processed and analyzed by artificial and computer to extract the difference information between images accurately in time. It is an important technical means in remote sensing information processing and application (Tang, 2013). The research of change detection mainly takes the aspects of image preprocessing, feature selection and extraction, threshold selection and so on. Early studies were based on lowand medium-resolution remote sensing images, which were based on pixel level and single spectral features. In recent years, with the continuous improvement of spatial resolution of remote sensing images, the details of ground objects are more abundant, and higher requirements for the method of change detection are put forward (Blaschke, 2010).

A variety of features produced by remote sensing images are the main carriers of the change information, including spectra, shapes, textures and other features. Conventional change detection uses a single feature as the research object. For example, Wei et al. proposed an adaptive algorithm based on the spectral feature fusion difference and ratio image according to the change of pixel gray value. In addition, index, edge and texture features show different advantages as single features, such as vegetation index characteristics are more sensitive to vegetation detection; vegetation index is more sensitive to vegetation detection; gradient reflects the degree of gray level changes of adjacent pixels in the region; texture reflects the spatial structure of objects by describing the image gray distribution. In addition, the multi-feature fusion algorithm has been proposed many times in recent years. For example, Du, P. et al. (2012) proposed the information fusion model of onedimensional and multi-dimensional eigenspaces, using the algorithm of fuzzy fusion to improve the integrity of the detected features. Difference statistics are used to describe the difference information between two temporals of the image, common methods are difference method, ratio method. For example, Lai, Z. et al. (2009) introduced the T-test method and the correlation coefficient method in probability statistics to extract the difference information. However, the gray values of the pixels in the map-spots mostly did not fit the Gaussian distribution. Using $\mathrm{T}$ test for statistical analysis, if not properly handled, it will have a larger deviation. Threshold selection in the process of change detection is crucial. In recent years, many scholars have proposed automatic threshold determination methods, such as KI automatic threshold selection method, EM automatic threshold method, MRFs automatic threshold method, but the actual application effect is not ideal. Although the degree of automation obtained by collecting typical sample features is lower than that of the above method, the accuracy of the method is maintained. In order to avoid the negative influence of atypical samples on the change detection results, a fuzzy evaluation function was introduced to determine the final change area.

\section{PRINCIPLE AND ALGORITHM}

The method proposed in this paper takes the map-spots as the basic unit of analysis, uses the improved Levene-Test to get the changed candidate map-spots, extracts the hue information, combines the texture features to carry on the analysis of the variation vector, and collects typical samples and determine the optimal threshold by the iterative method. According to the membership function, the membership degree of candidate mapspots are calculated to determine the final change region. Its specific procedures are:

1. It got map-spots by segmenting two overlapping images which had been pretreated, which is used as the basic research object to extracted features such as spectrum and texture. 
The improved Levene-Test was carried out with image gray value on each map-spots. Firstly, the original image data is transformed to reduce the relative radiation difference between the two images, and then the statistical information of the interclass/intra- class difference information is calculated to determine whether the difference between the two images is significant and determine the candidate region.

2. Hue information is extracted through the IHS Transform and conducts change vector analysis combined with the weighted texture information to obtain the change intensity information of each map-spot. A typical sample is selected to determine the optimal threshold of change by iteration, and the appropriate membership function is selected to calculate the membership degree of each map-spot and determine the final change region.

The technical process is shown in Figure 1.

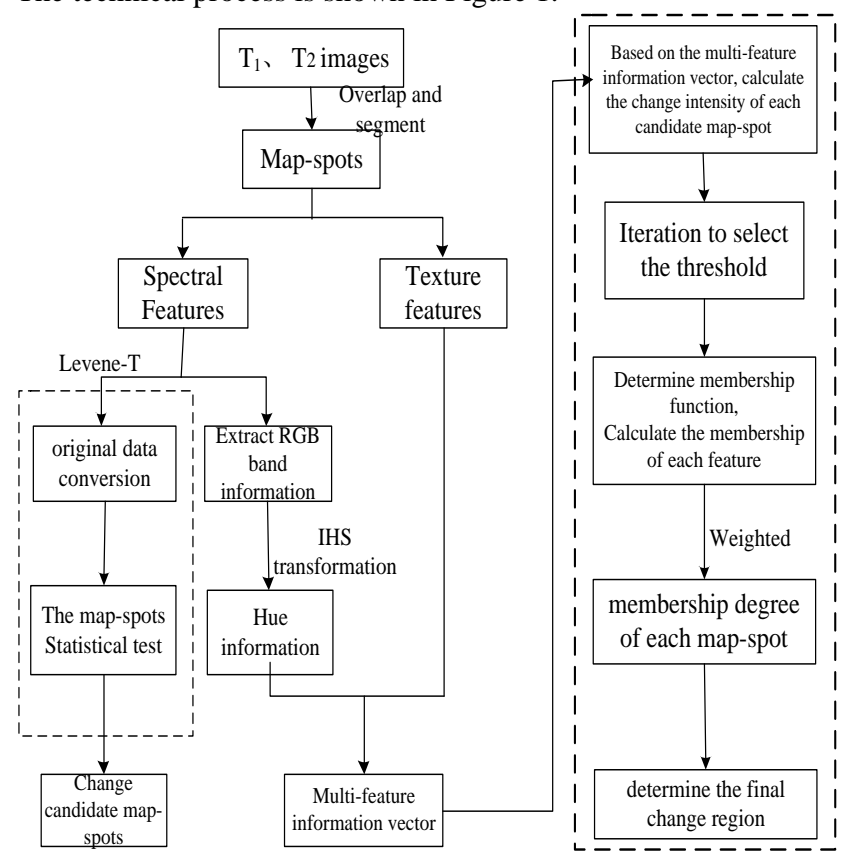

Figure 1. Technical Flow Chart

\subsection{Improved Levene-Test}

High-resolution remote sensing images provide rich detail information of ground objects, and the large amount of data and the more diversified distribution types undoubtedly increase the difficulty of data processing. In addition, due to the influence of imaging conditions, there are different degrees of radiation differences between the original image data. Even in the pretreatment stage after a rigorous radiation correction, there are still discrete pixels with large radiation differences in the mapspot, which are common in the type of artificial objects, and the use of conventional difference method will result in a certain false detection. In this paper, we proposed an improved LeveneTest to solve this problem, and to determine the change candidate region and reduce the subsequent calculation intensity.

The Levene-Test, proposed by H. Levene (1960), was expanded by M.B. Brown et al. (1974) and the diversification of the original data makes the Levene-Test more widely applicable. Levene-Test is essentially the one-way ANOVA after the transformation of the original data, and the main feature is that the sample is not involved in the comparison of the type of data distribution, which makes the Levene-Test more robust.
The improved Levene-Test takes the two corresponding mapspots as the research object, and statistically analyzes the gray information of the pixels in the two map-spots. Taking ZY-3 image data as an example, the test statistics are extracted from the gray information of the four bands in the map-spots to judge whether the difference between the two groups of data in the same band is significant, and the change candidate map-spots are comprehensively analyzed and determined. The gray information of each band in the two temporal is calculated, taking the first band as an example. The gray vectors (set as $U_{1}, U_{2}$ ) are as follows:

$$
\begin{aligned}
& U_{1}=\left\{X_{11}, X_{12}, \cdots, X_{1 n}\right\}^{\mathrm{T}} \\
& U_{2}=\left\{X_{21}, X_{22}, \cdots, X_{2 n}\right\}^{\mathrm{T}}
\end{aligned}
$$

Where $n$ represents the number of pixels in the map-spots. 1. The original data conversion: in the original two images the image gray value $X_{i j}$ is converted to the absolute value of the deviation $Y_{i j}(i=1,2 ; j=1,2, \cdots, n)$.

$$
Y_{i j}=\left|X_{i j}-M_{i \square}\right|
$$

Where $\mathrm{M}$ can be the arithmetic mean, median or adjusted mean of a patch in phase $i$, which is suitable for date of symmetric distribution, normal distribution and extreme discrete values. Therefore, Levene test has better robustness. In this paper, we use the data conversion method that adjusts the mean value to remove the discrete points with larger relative radiometric difference in the map-spot, and use the pixel gray mean value in the range $\mu \pm 2 \sigma$ as the adjustment mean value

$M_{i \square}$. Namely:

$$
M_{i \square}=\frac{1}{m} \sum X_{i j}
$$

Where $\mathrm{x}$ satisfies the condition: $\left|X_{i j}-\overline{X_{i \complement}}\right| \leq 2 \sigma, m$ is the number of pixels that satisfy the condition.

After the data conversion gray vector (set as $\boldsymbol{V}_{1}, \boldsymbol{V}_{2}$ ) are:

$$
\begin{aligned}
& V_{1}=\left\{\begin{array}{ll}
Y_{11}, Y_{12}, \cdots, & Y_{1 n}
\end{array}\right\}^{\mathrm{T}} \\
& V_{2}=\left\{\begin{array}{ll}
Y_{21}, Y_{22}, \cdots, & Y_{2 n}
\end{array}\right\}^{\mathrm{T}}
\end{aligned}
$$

After the original data is transformed, the relative radiation difference of the corresponding map-spot is greatly weakened. On the basis of this, the statistics of inter-class / intra-class data are carried out, which increases the credibility of the data difference information.

2. Calculate the test statistic w:

$$
W=\frac{(2 n-2) n\left[\left(\overline{Y_{1 \square}}-\overline{Y_{\square}}\right)^{2}+\left(\overline{Y_{2 \square}}-\overline{Y_{\square}}\right)^{2}\right]}{\sum_{j=1}^{n}\left[\left(Y_{1 j}-\overline{Y_{1 \square}}\right)^{2}+\left(Y_{2 j}-\overline{Y_{2 \square}}\right)^{2}\right]}
$$


Where $\overline{Y_{1 \square}}$ and $\overline{Y_{2 \square}}$ denote the mean values of each element in vectors $V_{1}$ and $V_{2} ; \bar{Y}_{\square}$ is the mean value of each element in $V_{1}$ and $V_{2}$ vectors.

3. The test statistic $W$ obeys the $F$ distribution with degree of freedom $v_{1}=1, \quad v_{2}=2 n-2$ under $\alpha$ level, and controls the intensity of change detection by adjusting the value of $\alpha$.

If $W \geq F(\alpha, 1,2 n-2), \quad P \leq \alpha$ reject $H_{0}$ at the level $\alpha$, the difference between the two groups is considered to be significant, which will be recognized as a candidate for change map-spot. Otherwise, it is considered that the difference between the two groups are not significant.

\subsection{Hue - texture feature extraction}

Each feature has its own unique texture information. Using the texture features to describe the difference information can not only make full use of the spectral information of the remote sensing image, but also solve the problem of the increase of the divisibility and the decrease of the reparability among classes in the high-resolution image. The commonly used texture description methods are based on statistical analysis, model method, structural method and mathematical transformation. The spatial gray level co-occurrence matrix proposed by Haralikc et al. (1979) is the most widely used method, which describes the Second-Order Statistics, and provides the information of the grayscale direction, the interval and the change range of the image, and extracts the statistics of texture features. In this paper, contrast, entropy and correlation are used to express the texture information of each map-spot.

Hue is the reflection of electromagnetic waves of surface objects on remote sensing images and is a characteristic of distinguishing colors from each other (Mei, A.,2001).The combination of different wavelengths in the visible spectral range stimulates the human eye to produce a colored sensation. The hue of the image is determined by the dominant wavelength of the reflected light, and various types of ground objects have their own dominant hue, which can distinguish these ground objects. High-resolution remote sensing images provide richer color information, and the dominant hue of the same objects are not uniform but tend to be stable, which provides a good theoretical basis for the extraction of the objects with different hue before and after the change (Zhang, Y., Li, L., Jiang, M.,et al., 2013).

Take the ZY-3 image data as an example, including four bands of blue, green, red and near infrared, and transform the two temporal images from the RGB system to the IHS system through the IHS transformation. IHS represents the color mode of hue, lightness and saturation, lightness indicates the brightness of the object, and saturation indicates the degree of color purity. Figure 2 shows the comparison of the effect of extracting the $\mathrm{H}$ component after the IHS transformation. By contrast, it can be found that the hue can better describe the difference information of the image.

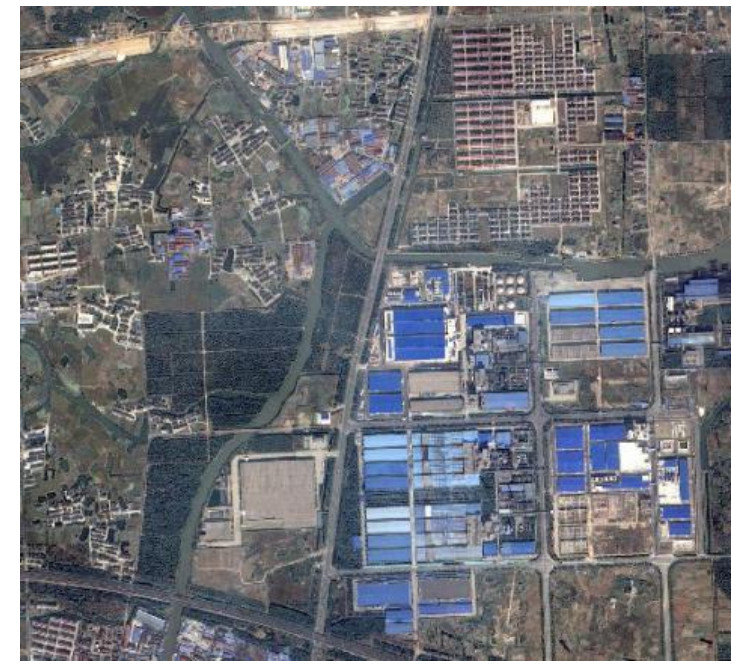

a) $\mathrm{T}_{1}$ Image

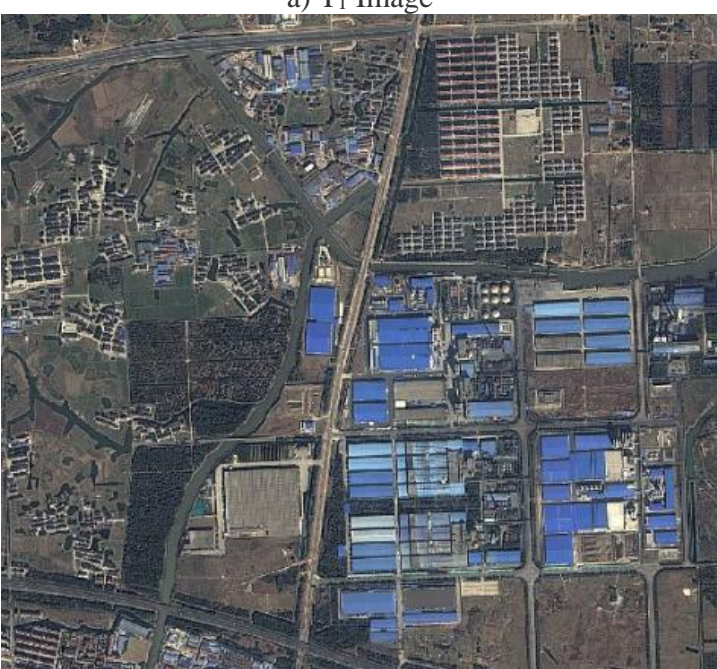

b) $\mathrm{T}_{2}$ Image

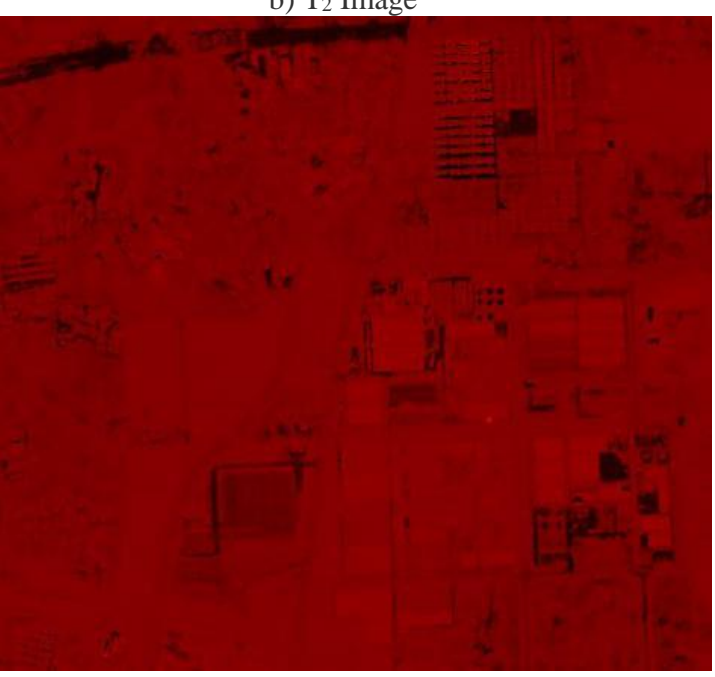

c) $\mathrm{H}$ Component of $\mathrm{T}_{1}$ Image 


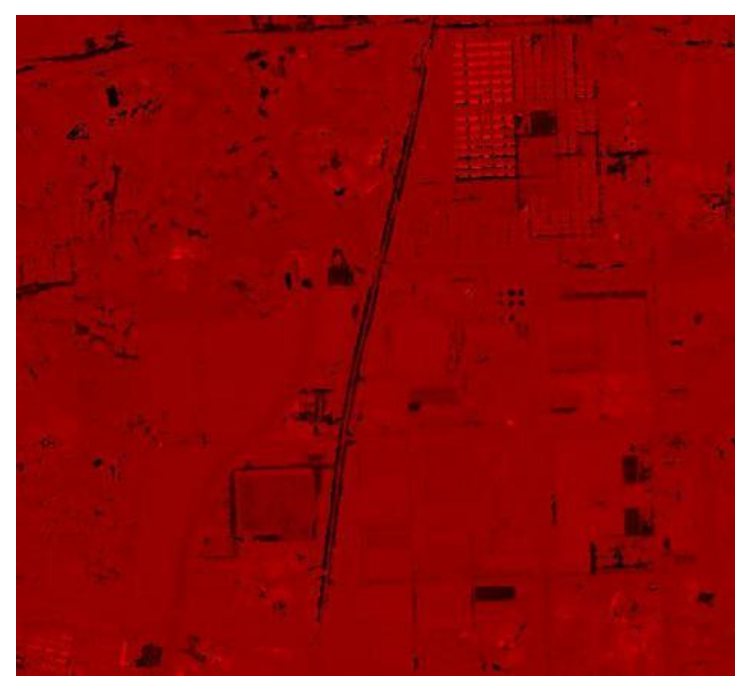

d) $\mathrm{H}$ Component of $\mathrm{T}_{2}$ Image

Figure 2. Effect Contrast of Two H Components after IHS Transform

\subsection{Change Vector Analysis and Threshold Selection}

The relative difference between the two images is calculated to obtain the change vector. The Euclidean distance of the change vector indicates the intensity of change. The Euclidean distance of the change vector indicates the intensity of change. The larger the value, the greater the probability of the change occurring. Taking the map-spot as the research object, the huetexture features are normalized to construct eigenvectors. Let the two-temporal eigenvectors of a map-spot be $A=\left(a_{1}, a_{2}, a_{3}, a_{4}\right)^{T}$ and $B=\left(b_{1}, b_{2}, b_{3}, b_{4}\right)^{T}$, where the components represent hue, contrast, entropy and correlation respectively.

$$
\Delta C=\left(a_{1}-b_{1}, a_{2}-b_{2}, a_{3}-b_{3}, a_{4}-b_{4}\right)^{T}
$$

Calculate the intensity of change:

$$
S=\sqrt{\lambda_{1}\left(a_{1}-b_{1}\right)^{2}+\lambda_{2}\left(a_{2}-b_{2}\right)^{2}+\lambda_{3}\left(a_{3}-b_{3}\right)^{2}+\lambda_{4}\left(a_{4}-b_{4}\right)^{2}}
$$

The magnitude of the intensity of change describes the degree of difference between the two map-spots of information, and weights each component by adjusting the size of the value. In order to ensure the reliability of the experiment, a certain number of typical samples were collected from the images with known ground change types, and the change threshold was determined by iterative analysis.

1. The mean $S$ of the intensity of the change of each map-spot is calculated as the initial threshold $T_{0}$.

2. Set the iteration increment $\Delta T$ and the termination value $T_{\text {end }}$, statistics the binary classification confusion matrix under a given threshold, and calculate the correct rate, the missed detection rate and the false detection rate.

3. After comprehensive comparison, the threshold with the highest detection accuracy is chosen as the optimal threshold $T_{\text {best }}$.

\subsection{Membership function determination and fuzzy evaluation}

The difference between different image data is large. Different features respond differently to the difference information. The influence of many factors often results in inconsistency of the test results. Fuzzy comprehensive evaluation is an effective multi-factor decision-making method for making a comprehensive evaluation of things affected by many factors. Fuzzy comprehensive evaluation is an effective multi-factor decision-making method to make a comprehensive evaluation of the things affected by many factors, the characteristic of which is that the evaluation result is not absolutely affirmative or negative, but is expressed by a fuzzy set (Metternicht, G. 1999.).Fuzzy set theory is used to describe uncertain elements and membership is used to describe the specific elements in the event.it is often used in data fusion and classification in the change detection to reduce the inconsistency of data.

In this paper, the optimal threshold is determined by collecting samples. In order to reduce the interference of the atypical samples in the sampling process, the fuzzy evaluation function is used to describe the membership of each feature and determine whether a certain map-spot has changed. The determination of membership function has no fixed form, and its function type is often determined according to a fuzzy concept. To a certain extent, the fuzziness of information is more accurately described, making the decision more reasonable. The commonly used fuzzy distributions are trapezoidal, K parabolic, normal, Cauchy, ridge and so on, each of which can be divided into three forms: Upper Limit, middle limit and lower limit. According to the characteristics of the membership degree of each feature, Partial Large-scale Distribution, Rising Ridge Distribution and S Distribution can be used. The specific form is shown in Figure 3.
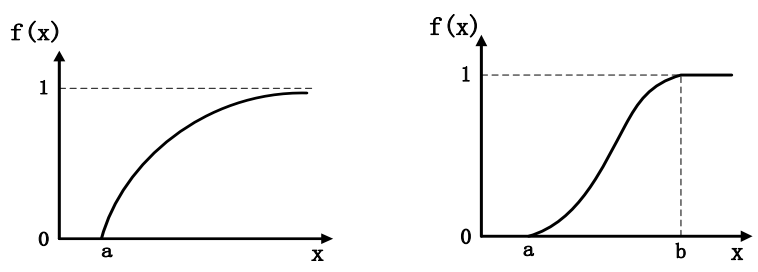

(a)Partial Large-scale Distribution (b) Rising Ridge Distribution

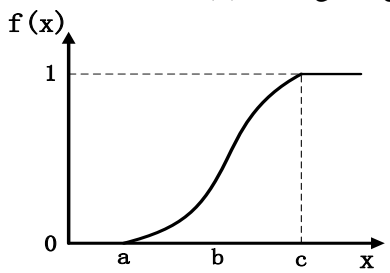

(c) S Distribution

Figure 3. Common Modalities of Fuzzy Distribution

In this paper, S-membership function is used for fuzzy evaluation. S-membership function can be used to describe most of the fuzzy concepts. It is described by a function whose shape is easy to adjust. As the independent variable increases, the membership degree increases .Let $\mathrm{S}$ denote the membership function that determines whether or not the map-spot has changed from the change intensity in the kth feature. If the change intensity is below a certain limit, it is assumed that there is no change in the map-spot. As the change intensity increases, the probability of changes of the map-spot increases until the map-spot is changed beyond a reasonable limit. 


$$
\phi\left(s_{k} ; \alpha, \beta, \mu\right)= \begin{cases}0 & s_{k} \leq \alpha \\ m\left(\frac{s_{k}-\alpha}{\mu-\alpha}\right)^{2} & \alpha<s_{k} \leq \beta \\ 1-n\left(\frac{s_{k}-\mu}{\mu-\alpha}\right)^{2} & \beta<s_{k}<\mu \\ 1 & s_{k} \geq \mu\end{cases}
$$

Where: The parameters $\boldsymbol{\mu}=\boldsymbol{T}_{\text {best }}, m 、 n$ often take 0.5 , by adjusting the parameters of the function $\mathrm{c}, \mathrm{d}$ to adapt to the actual data distribution. The membership degree of each feature is calculated, and the membership degree of the final change is determined by weighting, and the map-spot is determined to be a change / non-change class, and the final change region is extracted.

\section{TEST METHODS AND RESULTS}

In this paper, the experimental data are fusion images of ZY-3 in October 2013 and February 2015 in Jiangsu Province. The spatial resolution of the image is 2.1 meters. The image size is 1474 pixels * 1355 pixels. Two temporal images of the object types are rich, mainly including cultivated land, waters, buildings, roads, etc. The fractal network evolutionary algorithm (FNEA) is used to segment the two overlapping images. After segmentation, there are 477 map-spots, as shown in Figure 4.The improved Levene-Test is used to read the gray value of each pixel in the map-spot, constructing the $W$ statistic and judging its difference at the level of $\alpha=10^{-5} .315$ change candidate map-spots are obtained, which account for $66 \%$ of the total map-spot number, as shown in Figure 5 (black part is change candidate area).

It can be seen from the figure that the candidate map-spots extracted by the improved Levene-Test basically include all the change map-spots, and it has good fault tolerance for nonchanged map-spots with large radiation difference. It is excluded from the candidate region and does not participate in subsequent testing to avoid its adverse effects on the test results. Figure 6 is a map-spot of relatively large difference in radiation. Because Levene-Test algorithm extracts difference information based on the pixel level and there are many false detection in the test results, the change detection based on the hue-texture information and the fuzzy evaluation is carried out after the change candidate region is determined, so as to get higher accuracy detection results.

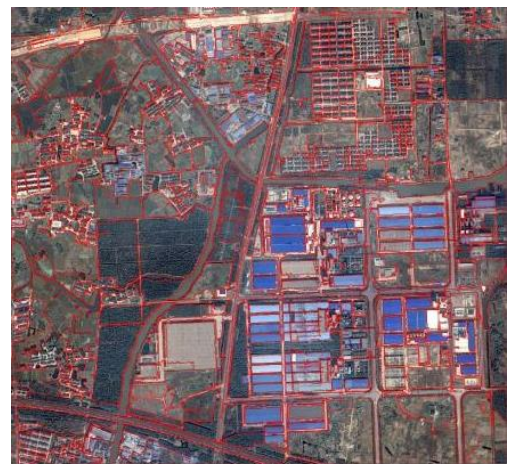

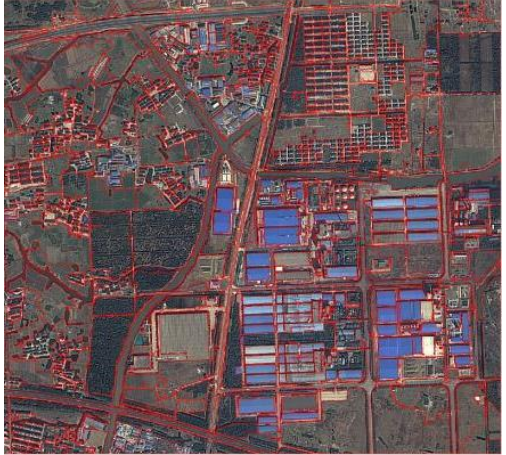

Figure 4. Segmentation Results of Two Overlapping Images

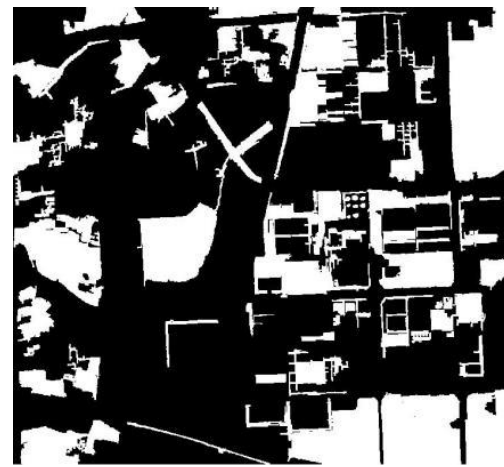

Figure 5. Candidate Change Regions Obtained by Improved Levene-Test (Dark Areas)

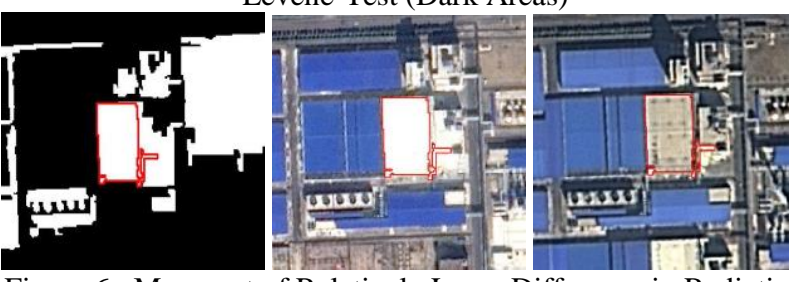

Figure 6. Map-spot of Relatively Large Difference in Radiation

In order to verify the effectiveness of the proposed method, different methods were used to monitor the changes in the test area, and the classification confusion matrix of each test result is calculated and the accuracy evaluation is carried out.

Erdas9.2 is used to extract the hue features after IHS transformation, and the texture information is represented by contrast, entropy and correlation. After normalization, the weight is $0.4,0.2,0.2,0.2$, and the intensity of each map-spot is calculated.

The typical sample was collected by iterative method to obtain the optimal threshold $T_{\text {best }}$ of 0.21 . The membership function was selected as $S$ distribution function, and the values of $\alpha, \beta$ and $\mu$ were $0.8 T_{b e s t}, 0.9 T_{\text {best }}$ and $T_{\text {best }}$ respectively. The membership degree of each feature is calculated, and the weights are $0.4,0.2,0.2$ and 0.2 respectively. Finally, the membership of each candidate map-spot is calculated to determine the final change region. Table 1 shows the comparison of the accuracy of different features with the decision method in the change detection. It can be seen that under the same decision method, the combination of hue and texture can better detect the change map-spot; under the same feature conditions, Fuzzy evaluation method has higher accuracy than direct threshold method. 


\begin{tabular}{|l|c|c|c|c|}
\hline & $\begin{array}{c}\text { Correct } \\
\text { rate }\end{array}$ & $\begin{array}{c}\text { Missing } \\
\text { detection } \\
\text { rate }\end{array}$ & $\begin{array}{c}\text { False } \\
\text { detectio } \\
\text { n rate }\end{array}$ & $\begin{array}{c}\text { Kappa } \\
\text { coeffici } \\
\text { ent }\end{array}$ \\
\hline Spectrum+Direct threshold & 0.36 & 0.62 & 0.4 & 0.31 \\
\hline Texture+Direct threshold & 0.4 & 0.52 & 0.44 & 0.38 \\
\hline $\begin{array}{l}\text { Hue-Texture+Direct } \\
\text { threshold }\end{array}$ & 0.52 & 0.16 & 0.38 & 0.60 \\
\hline $\begin{array}{l}\text { Spectrum + Fuzzy } \\
\text { Evaluation }\end{array}$ & 0.39 & 0.52 & 0.39 & 0.37 \\
\hline Texture+Fuzzy Evaluation & 0.45 & 0.52 & 0.36 & 0.41 \\
\hline $\begin{array}{l}\text { Hue-Texture+ Fuzzy } \\
\text { Evaluation }\end{array}$ & 0.81 & 0.09 & 0.25 & 0.84 \\
\hline
\end{tabular}

Table 1. Accuracy Comparison of Different Detection Methods As shown in Figure 7, through the manual interpretation of the reference change region, the change types mainly include: the road under construction turns into a completed road, and the non-artificial construction category becomes the artificial construction category. Figure 8 shows the detection results of each method, in which the white part is the change region of the detection. As can be seen from the figure: (a), (b) use a single feature and direct detection method, there are a large number of missed detection and false detection in the test results. The false detection is mainly concentrated in shadows, farmland and waters.(C) uses the hue-texture features and the direct threshold method, there are a large number of false detections and some missing detections in the test results, while the false detections mainly focus on farmland and watersheds.(d), (e) uses a single feature and fuzzy evaluation, there are a large number of false detections and some missing detections in the test results, while the false detections mainly focus on farmland and watersheds. (f) uses color-texture features and fuzzy evaluation, the detection accuracy is highest, the main change regions are detected, and there are very few missing and false detections.

In order to verify that Levene-Test can help to improve the final detection accuracy, The change map-spots obtained by combining the hue-texture features with the fuzzy evaluation count the accuracy of the change detection in the candidate map-spots and all map-spots respectively. The statistical results are shown in Table 2. It can be seen that using the Levene-Test to extract change candidate regions can greatly reduce the false detection rate and significantly improve the accuracy of the change detection.

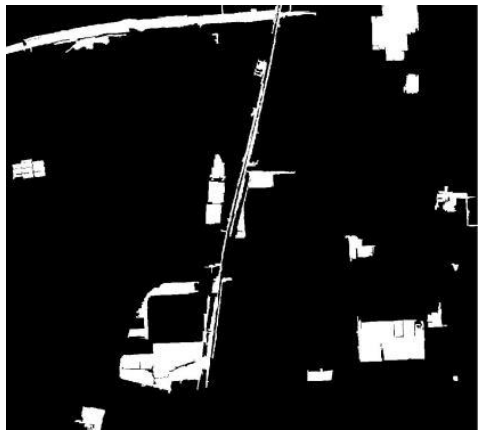

Figure 7. Reference of Change Regions
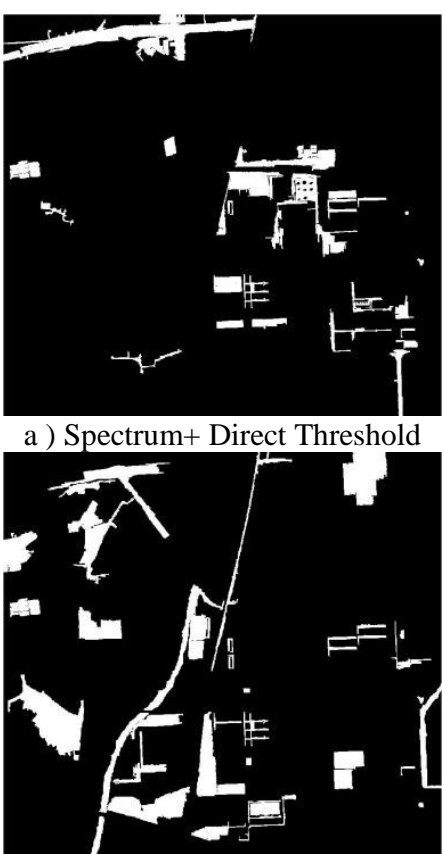

b ) Texture+ Direct Threshold

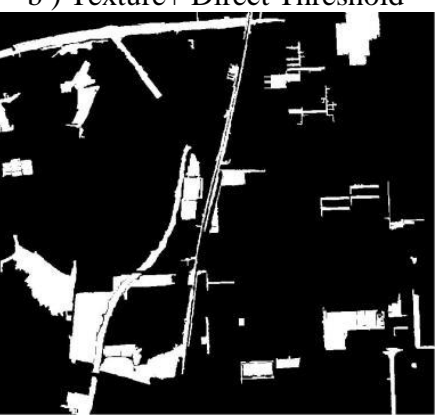

c ) Hue-Texture+ Direct Threshold
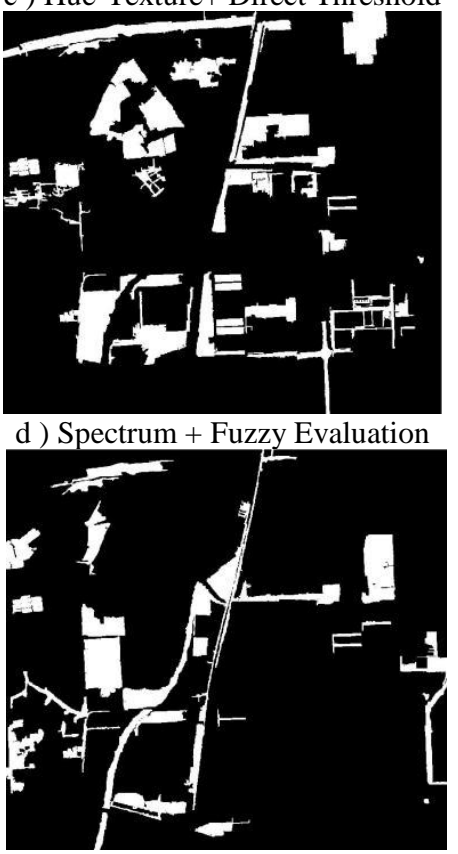

e) Texture+ Fuzzy Evaluation 


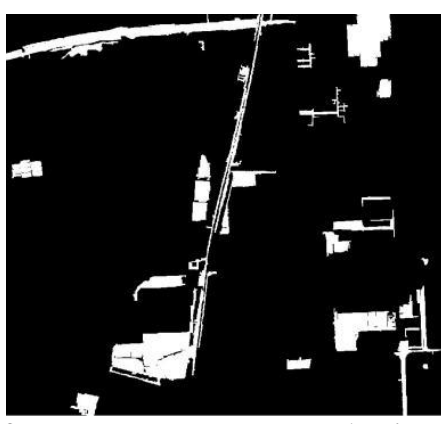

f) Hue-Texture+ Fuzzy Evaluation

Figure 8. Results of Each Detection Method

\begin{tabular}{|c|c|c|c|c|}
\hline & $\begin{array}{c}\text { Corr } \\
\text { ect } \\
\text { rate }\end{array}$ & $\begin{array}{c}\text { Missin } \\
\mathrm{g} \\
\text { detecti } \\
\text { on rate }\end{array}$ & $\begin{array}{c}\text { False } \\
\text { detecti } \\
\text { on rate }\end{array}$ & $\begin{array}{c}\text { Kappa } \\
\text { coeffic } \\
\text { ient }\end{array}$ \\
\hline $\begin{array}{l}\text { Hue-Texture+ Fuzzy } \\
\text { Evaluation (candidate } \\
\text { map-spots) }\end{array}$ & 0.36 & 0.62 & 0.4 & 0.31 \\
\hline $\begin{array}{c}\text { Hue-Texture+ Fuzzy } \\
\text { Evaluation (all map- } \\
\text { spots) }\end{array}$ & 0.81 & 0.09 & 0.25 & 0.84 \\
\hline
\end{tabular}

Table 2. Influence of Levene-Test on Detection Accuracy

\section{CONCLUSION}

This paper analyzes the shortcomings of traditional change detection and some advantages and disadvantages of the existing methods and proposes a remote sensing image change detection based on the Levene-Test and fuzzy evaluation. The image map-spots are obtained through the segmentation of the two temporal overlapping images. The spectrum, texture and hue of the map-spots are extracted. The candidate map-spots are obtained by the improved Levene-Test. The change vector analysis of hue-texture features is used to get the change intensity of each map-spot. The threshold value is determined by iterative method and the membership degree of the candidate map-spot is calculated to determine the final change region. The experimental results show that the algorithm proposed in this paper has a significant effect on the improvement of the detection accuracy. The conversion of the original data using the improved Levene-Test is very effective in reducing the influence of the map-spot of relatively large difference in radiation but not changing in reality. At the same time, the way to determine the change candidate map-spot greatly reduces the amount of subsequent calculations and improves the accuracy of the change detection. The application of hue-texture features and fuzzy evaluation significantly improved the results of change detection, There are few cases of missing detection and false detection, and the correct rate is obviously improved. In addition, there are still some shortcomings in this method. How to apply Levene-Test to more aspects of change detection and how to get the best threshold of change detection more quickly will be further studied and improved in the future.

\section{ACKNOWLEDGEMENTS}

The authors would like to express my gratitude to all those who helped me during the writing of this thesis. My deepest gratitude goes foremost to WenHui Chai, for her useful suggestions on my thesis. This research is fund by the National
Key Research and Development Program of China (NO.2016YFB0501403).

\section{REFERENCES}

Blaschke, T.,2010. Object based image analysis for remote sensing.ISPRS journal of photogrammetry and remote Sensing, 65(1),pp.2-16.

Brown, M. B., Forsythe A. B. 1974. Robust tests for the equality of variances. Journal of the American Statistical Association, 69(346),pp.364-367

Cao, Y. 2012. SAR image change detection algorithm, Xian Electronic and Science University,pp. 37-43.

Du, P., Liu, S.,2012. Change detection of remote sensing image by fusion of multiple features. Journal of Remote Sensing, 16(04), pp. 663-677.

Haralick, R.M.,1979. Statistical and structural approach to textures.Proceedings of IEEE, (67)5,pp.786-804.

Lai, Z., Shen, S., Cheng, X., et al. 2009. High-resolution remote sensing image change detection based on map-spot.Bulletin of Surveying and Mapping, (08),pp.17-20.

Levene, H. 1960. Robust tests for equality of variance. Contributions to Probability and Statistics: Essays in Honor of Harold Hotelling,pp.278-292.

Ma, G., Li, P., Qin, Q., 2006. Remote sensing image change detection based on fusion and generalized Gaussian model .Journal of Remote Sensing, 10(06),pp.847-853.

Mei, A.,2001. Introduction to Remote Sensing.Beijing: Higher Education Press, pp.85-87,120-122.

Metternicht, G. 1999. Change detection assessment using fuzzy sets and remotely sensing data: an application of topographic map revision.ISPRS journal of photogrammetry and remote Sensing, 54(4), pp.221-233.

Tang, Y.,2013. Object-oriented Multi-feature Change Detection in High-resolution Image in Cities, Wuhan : Wuhan University, pp.17-18 .

Wang, M., Zhang, J.,Li, H., et al. 2009. Research on highresolution remote sensing image change detection based on regional features .Surveying and Mapping Science, 34(1),pp.9294.

Wei, L., Zhong, Y., Zhang, L.,et al. 2010. Adaptive change detection of remote sensing image fusion. Journal of Remote Sensing, 14(6),pp.1196-1211.

Wu, K., Yang, R., Wang, Y.,et al . 2010. Research on multi spectral remote sensing image change detection based on PCA and EM algorithm. Computer Science, 37(03) ,pp.282-296.

Zhang, Y., Li, L., Jiang, M.,et al., 2013. A method of building change detection based on pixel level and feature level.Computer Science, 40(1),pp.286-293. 